\title{
CLIMA ORGANIZACIONAL EN UNA INSTITUCIÓN PRESTADORA DE SALUD DE MONTERÍA - CÓRDOBA, 2015
}

\author{
ORGANIZATIONAL CLIMATE IN A HEALTH PROVIDING INSTITUTION OF \\ MONTERIA - CORDOBA, 2015
}

\author{
Enalbis Esther Espitia C1, María Alejandra Cabrales $\mathrm{H}^{2}$, Katia Milena Morón $\mathrm{R}^{3}$ \\ Recibido para publicación: Octubre 4 de 2017 - Aprobado para publicación: Diciembre 6 de 2017
}

\begin{abstract}
RESUMEN
Objetivo: Determinar el clima organizacional de una Institución Prestadora de Salud (IPS), con el fin de establecer estrategias que permitan mejorar el ambiente laboral. Materiales y métodos: Estudio Descriptivo de corte transversal, con enfoque cuantitativo. La población la representaron el total de empleados de la institución, la muestra se aplicó una fórmula estadística que permitió establecer el total de 193 empleados a los que se les aplicó el instrumento de recolección de la información. Se utilizó la técnica encuesta mediante el instrumento cuestionario establecido por la Encuesta sobre el Clima Organizacional basado en los 4 pilares propuestos por la Organización Panamericana de Salud (OPS). Finalmente después de haber recolectado toda la información mediante las encuestas, se procedió a tabularlas mediante una hoja de Excel, y finalmente a realizar las tablas, gráficos y análisis correspondiente, según los objetivos y variables de estudio. De acuerdo a la resolución 08430 de 1993, el estudio fue clasificado sin riesgo, se respetó la confidencialidad, la anonimicidad del encuestado y la institución. Resultados. Se encontraron niveles de satisfacción por encima del $80 \%$ en factores como la comunicación, relaciones interpersonales y valores comunicativos y por debajo del $20 \%$ en factores como el bienestar social, nivel de capacitación y la compensación y reconocimiento. Conclusiones. Serealizóel diagnóstico del clima organizacional, el cual evidenció fortalezas en la comunicación, el trabajo en equipo, la coperación y liderazgo, por el contrario se obtuvierion oportunidad de mejora en factores como la promoción y el ascenso.
\end{abstract}

Palabras Clave: Clima organizacional, mejoramiento, motivación, comunicación.

\begin{abstract}
Objective. To determine the organizational climate of a Healthcare Provider Institution (HPI) to establish some strategies to improve the work environment. Methods and Materials. This was a quantitative, descriptive, and cross-sectional study. The population consisted of all the Institution's employees. A statistical formula was applied to choose the sample. This procedure allowed for the use of the data collection instrument with all the employees. A questionnaire for the survey established for the organizational climate based on the four pillars proposed by Health Pan-American Organization was used. Finally, once all the information was obtained through the survey, the tabulation was done through an Excel spreadsheet, tables and graphics were created, and data analysis completed according to the study variables. In line with Resolution 08430, 1993, the study was classified as riskfree. Also, the confidentiality and anonymity of both the research participants and the Institution were respected. Results. Levels of satisfaction higher than $80 \%$ were found in factors, such as communication, interpersonal relationships, and communicative values. Levels lower than $20 \%$ were found in factors, such as wellbeing, level of training as well as compensation and recognition. Conclusions. The diagnosis of the organizational climate revealed communication strengths, teamwork, cooperation, and leadership. Improvement opportunities in factors, such as promotion and raise were discovered as well.
\end{abstract}

Keywords: Organizational climate, improvement, motivation, communication.

\footnotetext{
${ }^{1}$ Magíster en Administración de Organizaciones, enfermera, docente asistente del programa Administración en Salud, Facultad Ciencias de la Salud, Universidad de Córdoba, grupo de investigación SAMSO, enalbisespitia@correo.unicordoba.edu.co.

${ }^{2,3}$ Administradora en Salud. Departamento de Salud Pública, Universidad de Córdoba.
} 


\section{INTRODUCCIÓN}

El clima organizacional es la percepción que cada uno de los empleados tienen acerca de cómo es el ambiente y el entorno en donde no solamente desarrollan sus actividades laborales, sinoendondetambiénse desarrollan relaciones dentro de sus grupo de trabajo, en donde se presentan distintos criterios, formas de pensar, personalidades lo que propende por fomentar relaciones y opiniones distintas muchas de las cuales se adoptan como forma de vida dentro del grupo de trabajo y forman una cultura organización con base a esas costumbres. Las Instituciones Prestadoras de Salud (IPS), como la gran mayoría de las empresas, están en proceso permanente de mejoramiento $y$ cumplimiento de las exigencias normativas del sistema de calidad, que incluye entre otras la satisfacción del cliente externo e interno, con eficacia, calidad y oportunidad.

Dado lo anterior, una IPS es definida como toda institución en Colombia que ofrece servicios de atención en salud, para ello establece una estructura orgánica adecuada, establece procesos para su funcionamiento y requiere asignar recursos financieros, técnicos, logísticos y de talento humano. Entre los equipos de trabajo se establecen relaciones entre el personal de la institución y los clientes externos. Según Valle (1), el clima organizacional y la satisfacción laboral son relevantes en el comportamiento del personal que labora en los hospitales; esto "potencia aspectos como la eficacia, diferenciación, innovación y adaptación...”. Por ello, e interesados en conocer cuál es el clima organizacionalenuna IPS, se realizóel presente estudio, con el fin de describir el estado actual con respectoal clima organizacionaly proponer estrategias de mejoramiento para la entidad.

\section{MATERIALES Y MÉTODOS}

Se realizó un estudio descriptivo de corte transversal, con enfoque cuantitativo, en el cual se incluyeron el total de empleados de la institución, cuyo número fue de 390 funcionarios, de los cuales 293 pertenecieron al área asistencial y 97 al área administrativa. Para obtener la muestra se aplicó una fórmula estadística que permitió establecer el total de 193 empleados a los que se les aplico el instrumento de recolección de la información.

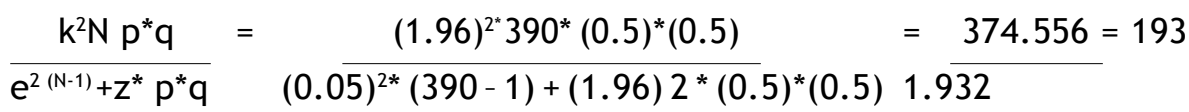

Para la recolección de la información se utilizó la técnica encuesta mediante el instrumento cuestionario diseñado por los investigadores y aprobado por la Jefe de Talento Humano y Jefe de Calidad de la institución, teniendo en cuenta y fusionando las variables de acuerdo con los objetivos planteados y lo establecido por la Encuesta sobre el Clima Organizacional basado en los 4 pilares propuestos por la Organización Panamericana de Salud (OPS) y la encuesta clima organizacional aportada por la empresa.
Este cuestionario consta de dos partes, la primera en la que se hace la identificación del área, se describe el objetivo y las instrucciones de diligenciamiento, y la segunda parte en la que se consideran en la columna de la derecha los cuestionamientos a los que el encuestado, de acuerdo con su experiencia, opinión y criterio establecía su respuesta de acuerdo con la siguiente escala: 1 Nunca, 2 Casi Nunca, 3 Algunos Veces y finalmente 4 siempre. El cuestionario fue aplicado en forma física a los funcionarios del área asistencial y en línea a los del área administrativa durante una semana en las tres jornadas laborales (mañana, 
tarde y noche). Finalmente después de haber recolectado toda la información mediante las encuestas, se procedió a tabularlas mediante una hoja de excel y finalmente a realizar las tablas, gráficos y análisis correspondiente, según los objetivos y variables de estudio. De acuerdo a la Resolución $n^{\circ} 008430$ de 1993 por la cual se establecen las normas científicas, técnicas yadministrativas para la investigación en salud en Colombia, esta investigación se clasificó con riesgo mínimo, puesto que es una investigación documentalenlaquenoserealiza ninguna intervención intencionada de variables biológicas, fisiológicas psicológicas o sociales del individuo que participa en el estudio. Se mantuvo el principio de confidencialidad de la información y el anonimato de los encuestados participantes y de lainstitución.

\section{RESULTADOS}

Una vez recolectada la información sobre cada una de las variables objeto de estudio con respecto al clima organizacional en las diferentes áreas de la institución se obtuvieron los siguientes resultados:

Factor comunicación: En el presente estudio está considerada como el intercambio efectivo de información entre un grupo de personas (1).

Tabla 1. Posibilidad de participar en las soluciones ante dificultades con respecto a al trabajo.

\begin{tabular}{|l|r|r|}
\hline \multicolumn{1}{|c|}{ Frecuencia } & Número & \multicolumn{2}{c|}{ Porcentaje } \\
\hline Nunca & 4 & 2,1 \\
\hline Casi Nunca & 24 & 12,4 \\
\hline Algunas Veces & 113 & 58,5 \\
\hline Siempre & 52 & 26,9 \\
\hline Total & 193 & 100 \\
\hline
\end{tabular}

Fuente: Cuestionario de la investigación.
Como se observa la mayoría de los encuestados manifiestan que al presentarse dificultades con respecto a su trabajo en un $58,5 \%$, a veces tiene la oportunidad de participar en las soluciones, algunos manifiestan en un $26,9 \%$ que siempre, en un $12,4 \%$ que casi nunca y el $2,1 \%$ que nunca.

Tabla 2. Me siento escuchado y valorado por mi jefe cuando le expreso mis ideas y puntos de vista.

\begin{tabular}{|l|r|r|}
\hline \multicolumn{1}{|c|}{ Frecuencia } & Número & Frecuencia Relativa \\
\hline Nunca & 8 & 4,1 \\
\hline Casi Nunca & 20 & 10,4 \\
\hline Algunas Veces & 80 & 41,5 \\
\hline Siempre & 85 & 44,0 \\
\hline Total & 193 & 100 \\
\hline
\end{tabular}

Fuente: Cuestionario de la investigación.

El 41.5\% de los encuestados afirma sentirse escuchadosyvalorados por sus jefesal expresar sus ideas y puntos de vista solo algunas veces, el $44.0 \%$ afirma siempre sentirse valorados, el $10.4 \%$ afirma casi nunca sentirse valorado y el 4.1\% afirma nunca sentirse valorados por sus jefes. Esto nos indica que más del $85 \%$ de los encuestados afirma que en la mayoría de los casos se han sentido valorados y escuchados por su superior, lo que da muestras de un ambiente donde prima la comunicación efectiva entre las partes y el dialogo por parte de los coordinadores de acuerdo al área.

Tabla 3. Comunicación clara de los objetivos, funciones y tareas del trabajo.

\begin{tabular}{|l|r|r|}
\hline \multicolumn{1}{|c|}{ Frecuencia } & \multicolumn{1}{c|}{ Número } & Frecuencia Relativa \\
\hline Nunca & 3 & 1,6 \\
\hline Casi Nunca & 16 & 8,3 \\
\hline Algunas Veces & 68 & 35,2 \\
\hline Siempre & 106 & 54,9 \\
\hline Total & 193 & 100 \\
\hline
\end{tabular}

Fuente: Cuestionario de la investigación 
El 54.9\% de los encuestados afirmaron recibir siempre por parte de sus jefes comunicaciones claras delos objetivos, funciones y tareas desus respectivos trabajos; el $35.2 \%$ afirmo que este tipo de comunicación solo lo reciben algunas veces, el $8.3 \%$ afirmaron que casi nunca y el $1.6 \%$ dijo nunca recibir información clara. Relaciones Interpersonales: Está contemplada como la forma en la que cada persona se relaciona con sus semejantes dentro de un ámbito cualquiera (2).

Tabla 4. Trabaja en equipo.

\begin{tabular}{|l|r|r|}
\hline \multicolumn{1}{|c|}{ Frecuencia } & Número & Frecuencia Relativa \\
\hline Nunca & 3 & 1,6 \\
\hline Casi Nunca & 6 & 3,1 \\
\hline Algunas Veces & 68 & 35,2 \\
\hline Siempre & 116 & 60,1 \\
\hline Total & 193 & 100 \\
\hline
\end{tabular}

Fuente: Cuestionario de la investigación.

El $60.1 \%$ de los encuestados asegura que en su área siempre se trabaja en equipo, mientras que $35.2 \%$ de los encuestados afirma que algunas veces en su área se trabaja en equipo, el 3.1\% y el $1.6 \%$ de los encuestados afirma que casi nunca y nunca respectivamente se trabajaen equipo en suárea.

Tabla 5. La institución da a conocer a los trabajadores su estructura organizacional como objetivos, política, misión, visión.

\begin{tabular}{|l|r|r|}
\hline \multicolumn{1}{|c|}{ Frecuencia } & \multicolumn{1}{|c|}{ Número } & Frecuencia Relativa \\
\hline Nunca & 2 & 1,0 \\
\hline Casi Nunca & 8 & 4,1 \\
\hline Algunas Veces & 43 & 22,3 \\
\hline Siempre & 140 & 72,5 \\
\hline Total & 193 & 100 \\
\hline
\end{tabular}

Fuente: Cuestionario de la investigación.
El $72.5 \%$ de los encuestados aseguran que la empresa siempre da a conocer su estructura organizacional, solo un $22.3 \%$ aseguran que algunas veces lo da a conocer y un $4.1 \%$ y $1 \%$ respectivamente aseguran que casi nunca y nunca lo da a conocer. Bienestar social y nivel de capacitación: son los niveles de satisfacción y entrenamiento que posee cada persona con respecto a la labor que realiza.

Tabla 6. Las instalaciones y servicios (accesos, sanitarios, servicios generales, etc.) en el centro de trabajo son buenos.

\begin{tabular}{|l|r|r|}
\hline \multicolumn{1}{|c|}{ Frecuencia } & \multicolumn{1}{|c|}{ Número } & Frecuencia Relativa \\
\hline Nunca & 3 & 1,6 \\
\hline Casi Nunca & 15 & 7,8 \\
\hline Algunas Veces & 80 & 41,5 \\
\hline Siempre & 95 & 49,2 \\
\hline Total & 193 & 100 \\
\hline
\end{tabular}

Fuente: Cuestionario de la investigación.

Un 49.2\% considera que siempre las instalaciones y servicios en el centro de trabajo son buenos, el $41.5 \%$ piensa que solo algunas veces es bueno, el $7.8 \%$ dice que casi nunca es bueno y un $1.6 \%$ dice que nunca es bueno.

Tabla 7. La empresa realiza jornadas de salud, recreación y deportes en donde integra a su grupo familiar.

\begin{tabular}{|l|r|r|}
\hline \multicolumn{1}{|c|}{ Frecuencia } & \multicolumn{1}{|c|}{ Número } & Frecuencia Relativa \\
\hline Nunca & 30 & 15,5 \\
\hline Casi Nunca & 44 & 22,8 \\
\hline Algunas Veces & 69 & 35,8 \\
\hline Siempre & 50 & 25,9 \\
\hline Total & 193 & 100 \\
\hline
\end{tabular}

Fuente: Cuestionario de la investigación. 
El 25.9\% considera que la empresa siempre realiza jornadas de salud, recreación y deportes en donde integra al grupo familiar, el $35.8 \%$ piensa que solo algunas veces lo hace, el $22.8 \%$ cree que casi nunca lo hace y el $15.5 \%$ considera que nunca lo hace. Compensación y reconocimiento: Son las retribuciones y reconocimientos ofrecidos a cada funcionario por el desempeño al que tiene lugar al realizar su labor (4).

Tabla 8. Las herramientas y los equipos con los que realiza su trabajo están en buen estado.

\begin{tabular}{|l|r|r|}
\hline \multicolumn{1}{|c|}{ Frecuencia } & Número & Frecuencia Relativa \\
\hline Nunca & 6 & 3,1 \\
\hline Casi Nunca & 7 & 3,6 \\
\hline Algunas Veces & 82 & 42,5 \\
\hline Siempre & 98 & 50,8 \\
\hline Total & 193 & 100 \\
\hline
\end{tabular}

Fuente: Cuestionario de la investigación.

El $50.8 \%$ de los encuestados afirma que siempre las herramientas y los equipos con los que realiza su trabajo están en buen estado, un $42.5 \%$ afirma que solo algunas veces están en buen estado, el $3.61 \%$ dice que casi nunca están en buen estado y el 3.1\% dice que nunca se encuentran en buenestado.

Tabla 8. Su desempeño en el trabajo es reconocido.

\begin{tabular}{|l|r|r|}
\hline \multicolumn{1}{|c|}{ Frecuencia } & \multicolumn{1}{|c|}{ Número } & Frecuencia Relativa \\
\hline Nunca & 11 & 5,7 \\
\hline Casi Nunca & 35 & 18,1 \\
\hline Algunas Veces & 84 & 43,5 \\
\hline Siempre & 63 & 32,6 \\
\hline Total & 193 & 100 \\
\hline
\end{tabular}

Fuente: Cuestionario de la investigación.
El 32.6\% afirma siempre ser reconocido su desempeño, el $43.05 \%$ dice que solo algunas veces es reconocido su desempeño en el trabajo, el $18.1 \%$ dice que casi nunca es reconocido su desempeño en el trabajo y el $5.7 \%$ afirma que nunca es reconocidosu desempeñoenel trabajo.

Tabla 9. Los empleados que tienen un desempeño sobresaliente son reconocidos.

\begin{tabular}{|l|r|r|}
\hline \multicolumn{1}{|c|}{ Frecuencia } & \multicolumn{1}{c|}{ Número } & Frecuencia Relativa \\
\hline Nunca & 23 & 11,9 \\
\hline Casi Nunca & 36 & 18,7 \\
\hline Algunas Veces & 80 & 41,5 \\
\hline Siempre & 54 & 28,0 \\
\hline Total & 193 & 100 \\
\hline
\end{tabular}

Fuente: Cuestionario de la investigación.

El 28.0\% de los empleados encuestados afirman ser siempre reconocidos cuando tienen un desempeño sobresaliente, el $41.5 \%$ dice que solo algunas veces son reconocidos, un $18.7 \%$ aseguran que casi nunca son reconocidos y el $11.9 \%$ dice que nunca son reconocidos.

Tabla 10. En su área se tiene en cuenta su opinión para el proceso de toma de decisiones.

\begin{tabular}{|l|r|r|}
\hline \multicolumn{1}{|c|}{ Frecuencia } & \multicolumn{1}{c|}{ Número } & Frecuencia Relativa \\
\hline Nunca & 25 & 13,0 \\
\hline Casi Nunca & 36 & 18,7 \\
\hline Algunas Veces & 102 & 52,8 \\
\hline Siempre & 30 & 15,5 \\
\hline Total & 193 & 100 \\
\hline
\end{tabular}

Fuente: Cuestionario de la investigación.

El 52.8\% afirma que algunas veces su opinión es tenida en cuenta en el proceso de toma de decisiones, un $15.5 \%$ dice que siempre y el porcentaje restante equivalente al $18.7 \%$ y $13.0 \%$ quienes dicen que casi nunca y nunca respectivamente. 


\section{DISCUSIÓN}

De acuerdo con Bédard y Chanlat, citados por Zapata(5), el clima organizacional es el "conjunto de suposiciones, creencias, valores y normas que comparten los miembros de una organización". De acuerdo con los resultados obtenidos, factores como la comunicación, el conocimiento de objetivos y tareas, las relaciones interpersonales, el trabajo en equipo, la cooperación mutua, actividades de bienestar social, capacitación, compensación y recompensa, presentan un buen nivel de aceptación en general con puntajes por encima del $80 \%$, lo cual es la percepción de los funcionarios comprometidos con la entidad, tal como lo manifiesta García(6), la "percepción que tiene el trabajador sobre la calidad de las relaciones entre él y los miembros de su equipo de trabajo, involucra en su percepción, el trato, el respeto, el apoyo, la comunicación y la empatía."

En otros factores como la promoción y el ascenso, programas de entrenamiento y desarrollo social con jornadas de recreación, remuneración y reconocimiento, y no tener en cuenta la opinión de los empleados en la toma de decisiones, obtuvieron los puntajes más bajos por debajo del $20 \%$ y se constituyen en una oportunidad de mejora para su intervención. El resultado es parecido con el obtenido en otros estudios como el de Aldana (7), en el que presenta una calificación de $60 \%$, lo que se convierte en un factor de intervención, ya que tal como lo afirma Del Río (8) "el involucramiento mental yemocional de los individuos en situaciones grupales que los estimula a contribuir a favor de las metas del grupo y a compartir la responsabilidad sobre ellas es un factor de motivación en el desempeño y la productividad".

Con respecto a la comunicación se pudo establecer que solo la cuarta parte de la población encuestada se siente involucrada de manera permanente en el desarrollo integral y efectivo de su trabajo, y más de la mitad de los encuestados afirman tener esta oportunidad de participación. Este resultado es similar al obtenido por Segredo (9), en el cual los trabajadores percibieron en un $60 \%$ como positiva con los medios y canales de comunicación formales e informales de la organización, los cuales facilitan el intercambio, orientan y fortalecen las relaciones entre las áreas y personas de la entidad.

Asímismo, se establece una similitud con el estudio realizado por Chiang, Salazar y Núñez (10), en el que realizan un análisis factorial para la escala de clima organizacional en 12 factores, de los cuales son comunes al presente estudio las variables de comunicación interna, reconocimiento, relaciones interpersonales en el trabajo, toma de decisiones, entorno físico, compromiso, adaptaciónal cambio, delegación de actividades y funciones, en el que se obtuvo un coeficiente alfa alto (entre 0,74 y 0,94 ).

La relación entre el clima organizacional y la satisfacción laboral se presenta en casi todas las dimensiones de clima organizacional: comunicación interna, conocimiento, relaciones interpersonales, calidad en el trabajo, toma de decisiones, objetivos de la institución, compromiso, adaptaciónal cambio, delegación de actividades y funciones, coordinación externa y eficiencia de la productividad, que tienen relaciones estadísticamente significativas con las siguientes variables de satisfacción laboral: satisfacción con la relación con los superiores, satisfacción con la participación en las decisiones y satisfacción con su trabajo en general. De igual forma, se encuentra similitud con los resultados en cuanto a la satisfacción de encuestados con relación al entorno físico, Chiang, establece una relación estadísticamente significativa con la variable de satisfacción laboral con las condiciones físicas de trabajo. 
La Escuela Nacional de Salud Pública en Cuba (11), tiene experiencia de aplicación en diferentes investigaciones de este instrumento propuesto por la OPS/OMS. Es de señalar que en los resultados obtenidos se demuestra que, el adecuado o inadecuado clima organizacional en las instituciones de salud repercute considerablemente en el desempeño de los profesionales y en la práctica de los directivos. Cada institución esúnica y tiene características propias que la diferencia de otras instituciones u organizaciones, por lo que resulta importante cuando se estudia el clima organizacional, explorar además de las dimensiones ya definidas por la OPS/OMS: liderazgo, motivación, retribución y participación, incluir la comunicación como otra de las dimensiones a estudiar $(9,10)$.

Este planteamiento tiene como base el reconocimiento de que la comunicación es una categoría sociopsicológica que expresa cómo se comportan las relaciones interpersonales, el compromiso ante las tareas y la calidad de la información, es decir, la comunicación debeser estudiada dentro de las dimensiones del clima sise tiene en cuenta que el trabajo del directivo transcurre en un proceso constante de recepción y transmisión de información, lo que supone una relación permanente de comunicación (11).

Dentro de las fortalezas de la institución, se destaca la motivación y compromiso con el trabajo de sus trabajadores y la satisfacción laboral con el trabajo que se realiza; el conocimiento de sus jefes jerárquicos; comprensión de la misión institucional; existen buenas relaciones interpersonales y suficiente flexibilidad para la supervisión de las tareas. En este contexto el difundir y hacer conocidas las políticas institucionales así como los valores que identifican la institución permitirán al personal involucrarse y participar tanto en el proceso cultural como en el proceso de cambio de la organización. Se evidencia además un ambiente donde prima la comunicación efectiva entre las partes y el dialogo por parte de los coordinadores deárea.

En cuanto a las debilidades, se mencionan la incapacidad de destacar y reconocer el trabajo bien hecho, la ausencia de una política de capacitación continua en el tiempo, el entorno físico inadecuado y el poco reconocimiento del trabajo bien hecho. Esto nos indica que la población encuestada no recibe con claridad la información respecto al plan de capacitación o no se motivan lo suficiente con respecto al logro de los objetivos, funciones y tareas lo que ocasiona grandes dificultades al momento de ejecutar los procedimientos, generando confusión y una brecha en la comunicación efectiva y la labor realizada.

El nuevo conocimiento que aporta el estudio, permite obtener, una visión rápida de que valoración tienen de la organización sus miembros, posibilitándole información a los directivosparalatoma dedecisionesylagestión del cambio. Sin embargo es necesario que se continúe ahondando en este tema y establecer la relación entre el clima y la satisfacción laboral en las instituciones de salud en Colombia, así mismo determinar el tipo de liderazgo ejercido y su percepción desde los trabajadores.

\section{CONCLUSIONES}

La comunicación la empresa tiene una fortaleza que debe aprovechar para impactar positivamente sobre las oportunidades de mejora y es el apoyo de los jefes inmediatos brindan a sus subalternos, estos manifiestan sentirse escuchados y valorados lo cual es ideal para mejorar los canales de comunicación, ya que actualmente no se informan oportunamente los cambios, así como temas que afecten directamente la estabilidad de los empleados, por otra parte se considera una oportunidad de mejora involucrar a los empleados en las soluciones de los problemas de su área, ya que de no hacerlo se genera frustración ya que 
la no calidad de la información los mantiene en un estado de inseguridad, desconfianza e indiferencia hacia los objetivos propuestos por la organización.

Dentro de la empresa en la categoría de las relaciones interpersonales se encontró una fortaleza en aspectos como el trato cordial, respetuoso y ameno entre los miembros de un equipo, lo cual permite controlar las situaciones adversas que se presenten en el día a día, aunque existan oportunidades de mejora que van desde la falta de compromiso y sentido de pertenencia hasta la falta de identidad en el grupo de trabajo, así como también se refleja la imposición de ideas lo que imposibilita la libertad de opinión, el trabajo es en grupo mas noenequipo, además deellola poca aceptación de responsabilidades por fallas evidenciadas, lo que ocasiona una brecha en la búsqueda del mejoramiento continuo para alcanzar la excelencia y la calidad de la atención en salud.

Existen dos fortalezas en la empresa que resaltan los valores de la misma ya que se esmera en que los empleados conozcan la plataformaestratégica locual hacequeestosse sienten orgullosos de pertenecer a la mismayse comprometan con su desarrollo y crecimiento. Entre las oportunidades de mejora tenemos las falencias en su infraestructura, importantes para brindar comodidad y confort en la realización de las tareas, desconocimiento de programas de capacitación y falta de flexibilidad para la actualización de los empleados, así como los cortos periodos de tiempo para el entrenamiento del personal nuevo, además de líderes que imprimen inseguridad y desconfianza entre los miembros de su equipo.

La reciprocidad y participación en la empresa tiene una fortaleza que consiste en brindar las herramientas y condiciones físicas adecuadas que le permiten a los empleados moverse en un entorno seguro, por otro lado tenemos las oportunidades de mejora donde la de mayor incidencia es la falta de reconocimiento y compensación por los resultados exitosos de un empleado, la inconformidad por la remuneración recibida, el desconocimiento sobre temas de salud y seguridad en el trabajo que se ve evidenciado ya que muchos desconocen temas de seguridad básicos debido a la falta de tiempo que genera su labor a pesar de las actividades que programa el comité paritario para dar conocimiento en estos temas.

La fluidez de la información genera un alto índice de oposición a los cambios presentados en la empresa, la falta de participación de los empleados en la solución a los problemas detectados en el desarrollo de los procesos y apatía a los eventos formales e informales programados por la institución.

La institución cuenta con un buen clima organizacional, la cual es direccionada desde la plataforma estratégica cimentada en el mejoramiento continuo, autocontrol, respeto trabajo en equipo y humanización del servicio, el recurso humano es idóneo, con vocación y espíritu de solidaridad comprometido con las metas de la empresa, enfocados hacia la excelencia y la calidad de la atención en salud.

\section{RECOMENDACIONES}

Conrespectoalacomunicación organizacional, con el fin de optimizar los canales de comunicación y fluidez de la información la utilización de notas informativas, folletos, reuniones semanales de cada área, actividades lúdicas entre los miembros de los equipos para fortalecer la empatía y cooperativismo, creación deunarevistaoperiódicoinstitucional que permita integrar las diferentes áreas y desarrollar iniciativas organizacionales.

Para fortalecer las relaciones interpersonales y fomentar el trabajo en equipo se deben practicar terapias de grupo operativo donde se puedan expresar opiniones personales, liberar tensiones 
y generar un ambiente de paz entre los equipos de trabajo, impulsar actividades recreativas como son: campeonatos deportivos de futbol, cicloruta, voleibol, entre otros, actividades de esparcimiento y entretenimiento, así como grupos de oración para encontrar equilibrio espiritual.

La empresa debe realizar actividades de reconocimiento y compensación que permitan a los empleados sentirse valorados y escuchados, donde se premien valores como la iniciativa, disciplina, responsabilidad, compromiso, respeto, sentido de pertenencia, tanto a nivel individual como en equipo, con lo cual semantendránmotivados loqueimpactara positivamente sobre los resultados del desempeño de las tareas y la productividad de la empresa, lo que se reflejara en la prestación del servicio y por ende en los índices de calidad de la institución. Finalmente se recomienda realizar la medición del clima organizacional en forma anual para el mejoramiento continuo.

\section{CONFLICTO DE INTERÉS}

Los autores declaran no tener conflicto de interés.

\section{REFERENCIAS}

1. Andrade H. Comunicación organizacional interna: proceso, disciplina y técnica. Netbiblo, 2005.

2. Sandoval Carameo MC. Concepto y dimensiones del clima organizacional. En Concepto y dimensiones del clima organizacional. Hitos de Ciencias Económico Administrativas (en línea) < http://www.ceaam.edu.mx/new/ae4/arh/ MOD_3_LECT_3.pdf> (citado en 15 de junio de 2015).

3. Mejía C. "Los valores corporativos." $L a$ estrategia del conocimiento. Ed Copyright Planning SA (2004).
4. Parker G, Jerry L. Cómo recompensar eficazmente a un equipo de trabajo: 27 Ejemplos actuales de Planes de Compensación y Reconocimiento de Equipos en Compañías líderes. Ediciones Díaz de Santos, 2013.

5. García S. Lo humano en la teoría de las organizaciones. En: VISIÓN GERENCIAL. no. 1 , p. $45-57$

6. García Solarte M.. Clima organizacional y su Diagnóstico: Una aproximación conceptual. En: Cuadernos De Administración. Vol. 25, No. 42, P. 43-61.

7. (7) Olga Ag. Clima Organizacional En Una Unidad De Segundo Nivel De Atención. En: Rev Enferm Inst Mex Seguro Soc. Vol. 17, no. 2, p. 91-96.

8. Del Rio-Mendoza J. Munares-Lovaton A y Montalvo-Valdez H. Clima Organizacional En Trabajadores De Un Hospital General De Ica, Peru. En: Revista Médica Panacea. Vol. 3, No. 1, P.11-14

9. Segredo Pérez Am. Clima Organizacional En La Gestión Del Cambio Para El Desarrollo De La Organización. En: Revista Cubana De Salud Pública. Vol. 39, no. 2 , p. 385-393

10. Chiang Vega, MM, Salazar Botello, CM, Núñez Partido, A. Clima organizacional y satisfacción laboral en un establecimiento de salud estatal: hospital tipo 1. Theoria [Internet]. 2007;16(2):61-76. Recuperado de: http://www.redalyc.org/articulo. oa?id=29916206

11. Segredo Pérez AM, Pérez Perea L. El Clima organizacional en el desarrollo de los Sistemas Organizativos. INFODIR. 2007[citado 4 Junio 2008];4. Disponible en: http://www.sld.cu/galerias/doc/sitios/ infodir /clima_organizacional_en_el_ 
desarrollo_de_sistemas_organizativos. doc.

12. MaríaCS.Conceptoydimensionesdelclia organizacional. En: HITOS DE CIENCIAS ECONÓMICO ADMINISTRATIVAS. Vol. 27, p. 7882.

13. Correa F. Los Factores Higiénicos De Frederick Herzberg No Son Suficientes Para Motivar.

14. López Más J. Motivación Laboral Y Gestión De Recursos Humanos En La Teoría De Frederick Herzberg. En: Gestión En El Tercer Milenio. Vol. 8, No. 15, P. $25-36$

15. López, Adriana. La Motivación. En: Recuperado Diciembre. Vol.24,

16. Márquez G. Área Académica: Administración Tema: Liderazgo Profesor (A): Mtra. Ivette Flores Jiménez Mtra. Ruth Flores Jiménez Lic. Iliana Tapia Castillo.
17. Rada C, Gómez A. Diseño, Construcción Y Validación De Un Instrumento Que Evalúa La Calidad De Vida La Boral Percibida En Organizaciones Colombianas, Desde La Teoría De Respuesta Al Item. En: Psychologia. Avances De La Disciplina. Vol. 4, No. 1, P. 113-124

18. Segredo Pérez A. Clima Organizacional En La Gestión Del Cambio Para El Desarrollo De La Organización. Rev Cubana Salud Pública [Internet]. 2013 Jun [citado 2018 Mayo 22]; 39( 2 ): 385-

393. Disponible en: http://scielo.sld.cu/ scielo.php?script=sci_arttext\&pid=S0864$34662013000200017 \& \operatorname{lng}=e s$.

19. Segredo Pérez AM. Clima organizacional en la gestión del coordinador docente de estado en la Misión Médica Cubana. República Bolivariana de Venezuela, 2009 [tesis]. La Habana: Escuela Nacional de Salud Pública; 2010.

20. Vargas Buitrago J. Propuesta De Mejoramiento Del Clima Laboral De La Alcaldia De Santa Rosa De Cabal. Universidad Tecnologica De Pereira. 2010. 